\title{
Application of GM(1,1) Model Based on Residual Error Correction in Athletic Performance Prediction
}

\author{
WANG Qing-bin ${ }^{1}$, JIA Ling 1 \\ 1.Zhuhai College of Jilin University, Zhuhai Guangdong, 519041 China
}

Keywords: Men's 200m Race; GM(1,1) Model; Residual Error

\begin{abstract}
In allusion to such problems in the athletic performance prediction as "poor information", "small sample" and "dynamics" difficult to solve through traditional statistical methods, the application of the grey model will more effectively and accurately solve the above problems. However, the application of the grey model in the athletic performance prediction also encounters many problems, for example: the traditional GM(1,1) model cannot obtain corresponding accuracy in many prediction problems. The application of $\operatorname{GM}(1,1)$ grey model based on residual error correction in the athletic performance prediction is researched in this article. Specifically, the best performances of the world men's 200m races during 2003 2013 are taken as the samples; then, $\mathrm{GM}(1,1)$ grey model based on residual error correction is adopted for sample modeling, wherein the prediction accuracy level of the model is the first level; then, the model obtained thereby is adopted for predicting the best performances of the world men's 200m race in 2014, and the predicted performance is 20.59s.
\end{abstract}

\section{Introduction}

In the past more than 30 years since Professor Deng Julong created grey mathematics in 1982, the application of grey mathematics and relevant theoretical research have had rapid and significant development, wherein the modeling fields involve decision-making, control, planning, prediction, etc., and the application fields thereof involve medical treatment, economy, education, sports, etc. Nevertheless, the application of grey model has more rapid development in the athletic field. According to the statistical information of relevant theses, the athletic theses with the grey model as the research method are increased every year at a speed of $9.81 \% \sim 10.10 \%$ in recent five years ${ }^{[1]}$. The rapid development of the application of the grey model in the athletic performance prediction has not only significantly improved the scientificity of the research on the athletic performance prediction, but also greatly promoted the continuous development of the grey mathematics theory. However, the grey model is sometimes abused in athletic field, and many problems not applicable to the grey model or not applicable to the traditional $\mathrm{GM}(1,1)$ grey prediction model are introduced into GM(1,1) grey model for modeling. Like this, the development of the grey mathematics theory and the application of the same in the athletic performance prediction will be undoubtedly influenced. At present, the application research on the grey model has become one of the most rapidly developed subjects in the athletic field. In this article, the performances of the world men's $200 \mathrm{~m}$ races are taken as the example to research the application of the grey model in the athletic performance prediction. Finally, GM(1,1) grey prediction model based on residual error correction is established through relevant research and analysis, thus not only providing reference for predicting the performances of men's 200m races in China, but also promoting the application and development of the grey model in the athletic field.

\section{GM(1,1) Model of the Best Performances of World Men's 500m Race}

Grey prediction is one of the main research directions in the grey theory, and the method application thereof is second only to the grey correlation analysis in the grey mathematics theory. $\operatorname{GM}(1,1)$ model has the features of simple method, wide application scope, high accuracy and 
strong practical applicability, and the application thereof has occupied $75.61 \%$ of all applications of the grey prediction model, and relevant theses are increased every year at a speed of $19.69 \%$ $21.08 \%$. The variation tendency of the best performances of the world men's $200 \mathrm{~m}$ races is analyzed in this article through relevant research, and meanwhile $\mathrm{GM}(1,1)$ model is applied to predict the development and change tendency thereof.

\section{Establishment of Residual Error Sequence GM (1,1) Model}

The accuracy of the prediction model is an important issue in the application of the grey model. In practical application, the accuracy of the grey prediction models including the athletic performance prediction model cannot meet corresponding requirement due to the data change and development characteristics, the model application, the knowledge quantity of the problem solver and other limitations. How to solve the prediction accuracy problem of the grey model and effectively improve and process the grey model becomes a major problem in the development of the grey model. It is known from the calculation result in the previous chapter that for the grey model established according to the best performances of the world men's $200 \mathrm{~m}$ races, the mean square error ratio test index thereof is 0.5289, and the accuracy level is the third level, namely: the prediction accuracy of the prediction model is relatively low. In allusion to the problem about how to improve the prediction accuracy of the grey model, many performance methods and theories have been proposed and most of them are mainly focused on processing the sample data and selecting the whitenization differential equation of the grey model. In order to improve the prediction accuracy of the best performances of the world men's 200m races, the residual error sequence grey prediction is implemented for this model to correct $\operatorname{GM}(1,1)$ grey prediction model.

\section{Establishment of Residual Error Sequence GM(1,1) Prediction Model}

Residual error refers to the difference between the predicted value of the grey prediction model and the actual value, and the residual error sequence grey prediction model refers to the improved prediction model obtained through firstly taking the residual error sequence as the original sequence of the grey prediction model for $\mathrm{GM}(1,1)$ modeling and then adopting the residual error prediction model obtained thereby to correct originally established $\mathrm{GM}(1,1)$ grey prediction model for improving the prediction accuracy of the prediction model. According to the above calculation, the residual errors of the best performances of the world men's 200m races are as shown in Table 1:

Table 1 Residual Errors of the Prediction Model of the Best Performances of

Men's 200m Races During 2003 2013

\begin{tabular}{ccccccc}
\hline Year & 2003 & 2004 & 2005 & 2006 & 2007 & 2008 \\
\hline Performance & 0 & -0.1542 & -0.0477 & 0.1688 & -0.1647 & 0.1618 \\
\hline Year & 2009 & 2010 & 2011 & 2012 & 2013 & \\
\hline Performance & 0.1883 & -0.0153 & 0.0612 & -0.0623 & -0.1358 & \\
\hline
\end{tabular}

The residual error sequence generated according to the residual errors of the best performances of the world men's 200m races during 2007 2013 is as follows:

$\varepsilon_{0}=\left(\varepsilon_{0}(1), \varepsilon_{0}(2), \cdots, \varepsilon_{0}(7)\right)$

According to Table 5, some residual errors are negative values and cannot be directly used for establishing GM(1,1) grey model. Meanwhile, for adopting these residual errors to establish the grey prediction model, the sequence class ratio $\lambda(k)$ thereof shall be within the interval of $\left(e^{-\frac{2}{n+1}}, e^{\frac{2}{n+2}}\right)$, or else, these residual errors shall not be used for establishing the grey prediction model. $n=7$ is put into $\left(e^{-\frac{2}{n+1}}, e^{\frac{2}{n+2}}\right)$ to obtain the interval $(0.7788,1.2488)$.

In order to establish $\mathrm{GM}(1,1)$ grey prediction model, the residual error sequence shall be processed to obtain the new sequence able to meet the grey model establishment conditions. In this article, the translation transformation is implemented for the residual error sequence $\varepsilon_{0}$, namely: take a suitable positive value $c$ to obtain $\varepsilon_{1}{ }^{(0)}(k)=\varepsilon_{0}(k)+c$. Here, $c=15$ is taken to obtain the 
converted sequence as shown in Table 2:

Table 2 New Residual Error Sequence

\begin{tabular}{ccccc}
\hline Year & 2007 & 2008 & 2009 & 2010 \\
\hline Performance & -0.1647 & 0.1618 & 0.1883 & -0.0153 \\
\hline Year & 2011 & 2012 & 2013 & \\
\hline Performance & 0.0612 & -0.0623 & -0.1358 & \\
\hline
\end{tabular}

According to the class ratio test, the class ratio scope of the new residual error sequence is [0.9785, 1.0136], and the establishment condition of $\operatorname{GM}(1,1)$ grey prediction model can be met. Afterwards, we can establish GM(1,1) grey prediction model according to the modeling steps.

\section{Result and Verification of Residual Error Sequence GM(1,1) Model}

$\mathrm{GM}(1,1)$ model is established for the new residual error sequence. The accumulative sequence thereof is set as $\varepsilon_{1}^{(1)}$ to obtain the solution of the whitenization differential equation thereof as follows:

$$
\hat{\varepsilon}_{1}^{(1)}(k+1)=3718.11-3703.27 \exp (-.00410962 k)
$$

The model error values are obtained according to relevant verification and the contrastive analysis of the predicted value and the original value, as shown in the following table:

Table 3 Error Values of New Residual Error Sequence GM(1,1) Model

\begin{tabular}{cccccc}
\hline Year & Original Value & Model Value & Residual Error & Relative Error & Class Ratio Difference \\
\hline 2007 & 14.8353 & 14.8353 & 0 & 0.0035 & \\
2008 & 15.1618 & 15.1878 & -0.0260 & 0.0002 & 0.0255 \\
2009 & 15.1883 & 15.1255 & 0.0628 & 0.0025 & 0.0058 \\
2010 & 14.9847 & 15.0635 & -0.0788 & 0.0079 & -0.0094 \\
2011 & 15.0612 & 15.0017 & 0.0595 & 0.0052 & 0.0092 \\
2012 & 14.9377 & 14.9402 & -0.0025 & 0.0088 & -0.0041 \\
2013 & 14.8642 & 14.8789 & -0.0147 & 0.0013 & -0.0008 \\
\hline
\end{tabular}

By virtue of the error values and the residual error values of the model in Table 3, residual error GM(1,1) model is calculated to obtain various verification indexes as shown in Table 4:

Table 4 Accuracy of Residual Error GM(1,1) Model

\begin{tabular}{cccc}
\hline Accuracy Level & Relative Error & Absolute Relevancy & Mean Square Error Ratio \\
\hline First Level & 0.0023 & 0.9978 & 0.3272 \\
\hline
\end{tabular}

According to Table 8 , the relative error of the model is 0.0023 , the accuracy level is the first level, the absolute relevancy is 0.9978 and the mean square error ratio is 0.3272 . Residual error $\mathrm{GM}(1,1)$ grey system has high accuracy for grey prediction model and can be used for residual error prediction.

For recovering the residual error sequence, the predicted values of the residual errors during 2003 2006 are set as 0 to obtain the predicted residual error values as shown in Table 5:

Table 5 Predicted Values of Residual Errors of the Best Performances of Men's 200m Races during 2003 2013

\begin{tabular}{ccccccc}
\hline Year & 2003 & 2004 & 2005 & 2006 & 2007 & 2008 \\
\hline Performance & 0 & 0 & 0 & 0 & -0.1647 & 0.1878 \\
\hline Year & 2009 & 2010 & 2011 & 2012 & 2013 & \\
\hline Performance & 0.1255 & 0.0635 & 0.0017 & -0.0598 & -0.1211 & \\
\hline
\end{tabular}

\section{Establishment of GM(1,1) Grey Prediction Model Based on Residual Error Correction}

According to relevant calculation in the previous chapter, the solution of the whitenization differential equation of the new residual error sequence is as follows: 


$$
\wedge(1)
$$

$$
\varepsilon_{1} \quad(k)=3718.11-3703.27 \exp (-.00410962 k)
$$

The solution of the above whitenization differential equation is obtained through adopting the sequence of the residual errors during 2007 2013 to establish GM(1,1) grey prediction model. In order to combine the solution with the result of $\operatorname{GM}(1,1)$ grey prediction model established according to the sequence of the best performances of the world men's $200 \mathrm{~m}$ races during 2003 2013, the above formula shall be processed.

$$
\varepsilon_{2}^{(1)}(k)= \begin{cases}0 & k \leq 4 \\ \varepsilon_{1}^{(1)}(k-4) & k>4\end{cases}
$$

If $y^{(0)}(k)(k=1,2, \cdots, 13)$ is assumed as the predicted value of $\operatorname{GM}(1,1)$ grey prediction model based on residual error correction, then $y^{(1)}(k)$ is the accumulative sequence of the predicted values of the residual error grey prediction model. Afterwards, the following formula can be obtained.

$$
y^{(1)}(k)=\hat{x}^{(1)}(k)+\varepsilon_{2}^{(1)}(k-4)
$$

The prediction sequence $y^{(0)}(k)$ is recovered through the accumulative sequence $y^{(1)}(k)$ in order to obtain the predicted values and the corresponding error values as shown in Table 6:

Table 6 Error Values of Grey Prediction Sequence GM(1,1) Model

\begin{tabular}{ccccc}
\hline Year & Original Value & Model Value & Residual Error & Relative Error \\
\hline 2003 & 20.13 & 21.3000 & 0 & 0 \\
2004 & 20.58 & 20.7342 & -0.1542 & 0.0075 \\
2005 & 20.69 & 20.7377 & -0.0477 & 0.0023 \\
2006 & 20.91 & 20.7412 & 0.1688 & 0.0081 \\
2007 & 20.58 & 20.5800 & 0 & 0 \\
2008 & 20.91 & 20.9360 & -0.0260 & 0.0012 \\
2009 & 20.94 & 20.8772 & 0.0628 & 0.0030 \\
2010 & 20.74 & 20.8188 & -0.0788 & 0.0038 \\
2011 & 20.82 & 20.7605 & 0.0595 & 0.0029 \\
2012 & 20.70 & 20.7025 & -0.0025 & 0.0001 \\
2013 & 20.63 & 20.6447 & -0.0147 & 0.0007 \\
\hline
\end{tabular}

By virtue of the error values and the residual error values of the model in Table 9, GM(1,1) grey prediction model based on residual error correction is calculated to obtain various verification indexes as shown in Table 7:

Table 7 Accuracy of GM(1,1) Prediction Model Based on Residual Error Correction

\begin{tabular}{cccc}
\hline Accuracy Level & Relative Error & Absolute Relevancy & Mean Square Error Ratio \\
\hline First Level & 0.0027 & 0.9980 & 0.3328 \\
\hline
\end{tabular}

According to Table 10, the relative error of the model is 0.0027 , the accuracy level is the first level, the absolute relevancy is 0.9980 and the mean square error ratio is 0.3328 . In other words, the prediction accuracy level of GM(1,1) grey prediction model based on residual error correction is the first level and this model can be used for accurately predicting the best performances of the world men's $200 \mathrm{~m}$ race.

Then, the above model is used to predict the best performances of the world men's $200 \mathrm{~m}$ race in 2014.

$$
\lambda^{(1)}(k+1)=122821 e^{-0.000168803 k}-122801
$$

According to the above formula, when $k=10$ and $k=11$ are true, we can $\operatorname{obtain}_{X}^{\wedge(1)}(11)=227.5006$ and ${ }_{X}^{\wedge(1)}(12)=248.2699$.

Namely, the predicted value obtained from $\mathrm{GM}(1,1)$ grey prediction model for 2004 
is $\hat{X}^{\wedge(0)}(12)=20.7639$.

Similarly, the predicted value of the residual error for 2014 is $\hat{\varepsilon}_{2}(8)=-0.1821$. Therefore, the best performance of the world men's $200 \mathrm{~m}$ race in 2014 is calculated as 20.59 according to GM(1,1) model based on residual error correction.

\section{Conclusion}

In this article, the grey prediction model is established according to the best performances of the world men's $200 \mathrm{~m}$ races in order to not only predict the performance of the world men's 200m race in 2014, but also discuss the application method of the grey model in the athletic performance prediction, thus promoting the application of the grey model in the athletic sports. $\mathrm{GM}(1,1)$ grey model is applicable to the modeling process with the sample data distribution presented in index movement. Through establishing relevant model for the statistical data for analysis, the grey prediction model is extended. Meanwhile, $\mathrm{GM}(1,1)$ grey prediction model based on residual error correction is applied to predict the best performance of the world men's 200m race in 2014, and this model is also compared with $\mathrm{GM}(1,1)$ grey model for contrastive analysis. According to the comparison result, we can find that $\operatorname{GM}(1,1)$ grey prediction model based on residual error correction has the features of low complexity and high prediction accuracy, thus applicable to the athletic performance prediction.

\section{Acknowledgement}

The zhuhai college of jilin university, one hundred engineering project funds

\section{References}

[1] Su T, Lv Z, Gao S, et al. 3d seabed: 3d modeling and visualization platform for the seabed[C]. Multimedia and Expo Workshops (ICMEW), 2014 IEEE International Conference on. IEEE, 2014: 1-6.

[2] Lu Z, Lal Khan M S, Ur Réhman S. Hand and foot gesture interaction for handheld devices[C]. Proceedings of the 21st ACM international conference on Multimedia. ACM, 2013: 621-624.

[3] Zhang $\mathrm{X}, \mathrm{Xu} \mathrm{Z}$, Henriquez $\mathrm{C}$, et al. Spike-based indirect training of a spiking neural network-controlled virtual insect[C]. Decision and Control (CDC), 2013 IEEE 52nd Annual Conference on. IEEE, 2013: 6798-6805.

[4] Dang S, Ju J, Matthews D, et al. Efficient solar power heating system based on lenticular condensation[C]. Information Science, Electronics and Electrical Engineering (ISEEE), 2014 International Conference on. IEEE, 2014, 2: 736-739. 